\title{
Effects of step length and cadence on hip moment impulse in the frontal plane during the stance phase
}

\author{
Takuma Inai $^{\text {Corresp., }}{ }^{,}$, Tomoya Takabayashi ${ }^{2}$, Mutsuaki Edama $^{2}$, Masayoshi Kubo ${ }^{2}$ \\ ${ }^{1}$ Exercise Motivation and Physical Function Augmentation Research Team, National Institute of Advanced Industrial Science and Technology, Kashiwa \\ City, Japan \\ 2 Institute for Human Movement and Medical Sciences, Niigata University of Health and Welfare, Niigata City, Japan \\ Corresponding Author: Takuma Inai \\ Email address: hwd17001@nuhw.ac.jp
}

\section{Background}

An excessive daily cumulative hip moment in the frontal plane (determined as the product of hip moment impulse in the frontal plane during the stance phase and mean number of steps per day) is a risk factor for the progression of hip osteoarthritis. Moreover, walking speed and step length decrease, whereas cadence increases in patients with hip osteoarthritis. However, the effects of step length and cadence on hip moment impulse in the frontal plane during the stance phase are not known. Therefore, this study aimed to examine the effects of step length and cadence on hip moment impulse in the frontal plane during the stance phase.

\section{Methods}

We used a public dataset (kinetic and kinematic data) of over-ground walking and selected 31 participants randomly from the full dataset of 57 participants. The selected participants walked at a selfselected speed and repeated the exercise 15 times. We analyzed the data for all 15 trials for each participant. Multiple regression analysis was performed with the hip moment impulse in the frontal plane during the stance phase as the dependent variable and step length and cadence as independent variables.

\section{Results}

The adjusted $R^{2}$ in this model was $0.71(p<0.001)$. The standardized partial regression coefficients of step length and cadence were $0.63(t=5.24 ; p<0.001)$ and $-0.60(t=-4.58 ; p<0.001)$, respectively.

\section{Conclusions}

Our results suggest that low cadence, not short step length, increases the hip moment impulse in the frontal plane. Our findings help understand the gait pattern with low hip moment impulse in the frontal plane. 
1 Effects of step length and cadence on hip moment 2 impulse in the frontal plane during the stance phase

3

4 Takuma Inai ${ }^{1}$, Tomoya Takabayashi ${ }^{2}$, Mutsuaki Edama ${ }^{2}$, Masayoshi Kubo ${ }^{2}$ 5

$6 \quad{ }^{1}$ Exercise Motivation and Physical Function Augmentation Research Team, National Institute of

7 Advanced Industrial Science and Technology, Kashiwa City, Chiba, Japan

$8{ }^{2}$ Institute for Human Movement and Medical Sciences, Niigata University of Health and

9 Welfare, Niigata City, Niigata, Japan

10

11 Corresponding Author:

12 Takuma Inai

13 Kashiwa II Campus, University of Tokyo,

14 6-2-3 Kashiwanoha, Kashiwa, Chiba 277-0882, JAPAN

15 Email address: hwd17001@nuhw.ac.jp 


\section{Abstract}

\section{Background}

18 An excessive daily cumulative hip moment in the frontal plane (determined as the product of hip 19 moment impulse in the frontal plane during the stance phase and mean number of steps per day) 20 is a risk factor for the progression of hip osteoarthritis. Moreover, walking speed and step length 21 decrease, whereas cadence increases in patients with hip osteoarthritis. However, the effects of 22 step length and cadence on hip moment impulse in the frontal plane during the stance phase are 23 not known. Therefore, this study aimed to examine the effects of step length and cadence on hip 24 moment impulse in the frontal plane during the stance phase.

\section{Methods}

26 We used a public dataset (kinetic and kinematic data) of over-ground walking and selected 31

27 participants randomly from the full dataset of 57 participants. The selected participants walked at 28 a self-selected speed and repeated the exercise 15 times. We analyzed the data for all 15 trials for 29 each participant. Multiple regression analysis was performed with the hip moment impulse in the 30 frontal plane during the stance phase as the dependent variable and step length and cadence as 31 independent variables.

\section{Results}

33 The adjusted $R^{2}$ in this model was $0.71(p<0.001)$. The standardized partial regression 34 coefficients of step length and cadence were $0.63(\mathrm{t}=5.24 ; p<0.001)$ and $-0.60(\mathrm{t}=-4.58$; $35 p<0.001)$, respectively.

\section{Conclusions}

37 Our results suggest that low cadence, not short step length, increases the hip moment impulse in 38 the frontal plane. Our findings help understand the gait pattern with low hip moment impulse in 39 the frontal plane. 
40

41

42

43

44

45

46

47

48

49

50

51

52

53

54

55

56

57

58

59

60

61

62

63

64

65

66

67

68

69

70

71

72

73

74

75

76

77

78

79

\section{Introduction}

Degeneration of the hip articular cartilage is noted in patients with hip osteoarthritis, which reduces their physical function ability. Patients with hip osteoarthritis experience hip joint pain (Iidaka et al., 2016, 2020), and decrease in muscle strength (Loureiro, Mills \& Barrett, 2013; Zacharias et al., 2016; Loureiro et al., 2018) and range of hip joint motion (Holla et al., $2011,2012)$. Such changes decrease the patients' ability to perform activities of daily living (Pisters et al., 2012, 2014) and reduces their quality of life (Salaffi et al., 2005; Boutron et al., 2008). It is, therefore, important to prevent the progression of hip osteoarthritis to avoid these problems.

Tateuchi et al. (2017) proposed a new index in the frontal plane called daily cumulative hip moment (calculated as the product of hip moment impulse in the frontal plane and mean number of steps per day) and examined the relationship between this index and the progression of hip osteoarthritis (i.e., width of the hip joint space). They found that an excessive daily cumulative hip moment in the frontal plane is a risk factor for narrowing of the hip joint space width (Tateuchi et al., 2017). Therefore, it is important to avoid excessive daily cumulative hip moment in the frontal plane to prevent narrowing of the hip joint space width.

Considering that the walking speed is lower in individuals with hip osteoarthritis than in those without hip osteoarthritis (Constantinou et al., 2017; Foucher, 2017; Meyer et al., 2018; Wesseling et al., 2018; Diamond et al., 2018), Inai et al. (Inai et al., 2019a) examined the relationship between walking speed and hip moment impulse in the frontal plane and found that a decrease in the walking speed increases the hip moment impulse in the frontal plane during the stance phase. Therefore, to avoid a high daily cumulative hip moment in the frontal plane, patients with hip osteoarthritis should maintain a normal walking speed (i.e., avoid reducing the walking speed).

Walking speed is calculated as the product of step length and cadence. Constantinou et al. (2017) found that step length decreases for patients with hip osteoarthritis compared to controls.

Schmidt et al. (2017) showed that cadence is greater in patients with hip osteoarthritis increases than in controls. However, the effects of step length and cadence on hip moment impulse in the frontal plane during the stance phase are not known.

This study aimed to examine the effects of step length and cadence on hip moment impulse in the frontal plane during the stance phase. We hypothesized that (1) a decrease in step length decreases the hip moment impulse in the frontal plane and (2) a decrease in cadence increases the hip moment impulse in the frontal plane. To the best of our knowledge, this is the first study to evaluate the effects of step length and cadence on hip moment impulse in the frontal plane.

\section{Materials \& Methods}

\section{Participants}

Data were collected as previously described in Horst et al. (2019a,b). Specifically, 57

healthy adults (29 women, 28 men; age: 23.1 (2.7) years; height: 1.74 (0.10) m; body mass: 67.9

Peer] reviewing PDF | (2020:11:55806:4:0:NEW 28 Jun 2021) 
80 (11.3) kg) participated. Prior to the experiment, each participant read and signed a consent form 81 that had previously been approved by the ethical committee of the Rhineland-Palatinate Medical 82 Association in Mainz, Germany (Horst et al., 2019a,b).

\section{Experimental protocol and data acquisition}

84 The details of the experimental protocol and data acquisition were described previously 85 (Horst et al., 2019a,b). All participants performed 20 walking trials (self-selected speed; 86 barefoot) on a 10-m path. Because some of the trials had technical errors, we used 15 trials for 87 each participant. Ten Oqus 310 infrared cameras (Qualisys AB, Sweden) captured the three88 dimensional marker trajectories at a sampling frequency of $250 \mathrm{~Hz}$. Three-dimensional ground reaction forces were recorded by two Kistler force plates (Type 9287CA - Kistler, Switzerland)

90

91

92

93

94

95

96

97

98

99

100

101

102

103

104

105

106

107

108

109

110

111

112

113

114

115

116

117

118 at a frequency of $1000 \mathrm{~Hz}$. A full-body marker set consisting of 62 reflective markers placed on anatomical landmarks was used. For the static trial, all 62 reflective markers were used. For the dynamic trial (i.e., walking trial), only 54 reflective markers were used. Two experienced assessors attached the markers and conducted the analysis.

\section{Data processing}

First, we conducted a statistical power analysis for linear regression analysis using $\mathrm{G}^{*}$ Power 3.1 software (effect size $\mathrm{f}^{2}=0.35$ [large] based on Cohen (1992), significance level = 0.05 , power $=0.8$, number of predictors $=0.2$, which indicated that the required sample size was 31 subjects. Therefore, we selected 31 participants randomly (15 women, 16 men; age: 23.0 [2.6] years; height: 1.76 [0.10] m; body mass: 68.8 [10.9] kg) from the 57 participants. For each participant, the data for 15 trials were randomly selected for analysis. We evaluated the stance phase of the right limb (i.e., from right heel contact to right toe-off) for each participant (Tateuchi et al., 2017). The three-dimensional marker trajectories, ground reaction forces, center of pressures, and moments of the force plates were filtered using a fourth-order Butterworth lowpass filter at a cut-off frequency of $6 \mathrm{~Hz}$ (Inai et al., 2019b).

The external hip adduction moment during the stance phase of the right limb was calculated for each participant using inverse dynamics (Newton-Euler method). Subsequently, the hip joint moment impulse in the frontal plane was calculated by integration of the external hip adduction moment. The hip moment impulse in the frontal plane (Nm s) was normalized by body mass $(\mathrm{Nm} \mathrm{s} / \mathrm{kg})$. To confirm the quantitative validity of the external hip adduction moment during the stance phase, we also calculated the first peak external hip adduction moment during the first half $(0-50 \%)$ of the stance phase. The first peak external hip adduction moment $(\mathrm{Nm})$ was normalized with body mass $(\mathrm{Nm} / \mathrm{kg})$.

Mass, mass position, and inertia parameters of the segments reported previously (de Leva, 1996; Robertson et al., 2013) were used. The hip joint center was derived as a point interpolated at a distance of $18 \%$ of the vector norm from each reflective marker of the superior aspect of the greater trochanter along the vector (Kito et al., 2010). The right knee joint center was defined as the midpoint of the medial and lateral epicondyles of the right femur. The right ankle joint center was defined as the midpoint of the right medial and lateral malleoli. 
119

120

121

122

123

124

125

126

127

128

129

130

131

132

133

134

135

136

137

138

139

140

141

142

143

144

145

146

147

148

149

150

151

152

153

154

155

156

157

158

The step length from the point of right heel contact was defined as the distance between the right and left heels. The step length was normalized with height $(\mathrm{m} / \mathrm{HT})$. Cadence (steps/min) was calculated using the time from right heel contact to left heel contact. Walking speed was calculated using the reflective markers placed on the sacrum and normalized with height (m/HT). Stance time was defined as the time from the time of right heel contact to that of right toe-off. All gait analyses were performed using Scilab (Scilab Enterprises, France).

\section{Statistical analysis}

Multiple regression analysis was performed in which the dependent variable was defined as hip moment impulse in the frontal plane during the stance phase, and the independent variables were defined as step length and cadence. To confirm the multicollinearity of this model, the variance inflation factor was calculated. We also confirmed whether the residuals of the model followed a normal distribution, and the correlation coefficient between step length and cadence was also confirmed.

The Shapiro-Wilk test was used to determine whether the variables (step length, cadence, walking speed, stance time, and hip moment impulse in the frontal plane) followed a normal distribution. The Pearson's correlation or Spearman's correlation was used, depending upon the results of the normality test. The significance level was set at $<0.05$.

\section{Results}

Table 1 shows the results of the multiple regression analysis. A positive relationship between step length and hip moment impulse in the frontal plane was observed (i.e., a decrease in step length decreased the hip moment impulse in the frontal plane). An inverse relationship between cadence and hip moment impulse in the frontal plane was also observed (i.e., a decrease in cadence increased the hip moment impulse in the frontal plane). The variance inflation factor in the multiple regression analysis was1.136, and the residuals followed a normal distribution $(p=0.905)$.

Figure 1 shows the relationship among step length, cadence, and hip moment impulse in the frontal plane. Long step length and low cadence resulted in an increased hip moment impulse in the frontal plane. In contrast, short step length and high cadence resulted in a decreased hip moment impulse in the frontal plane.

Figure 2 shows the waveform of the average external hip adduction moment during the stance phase $(\mathrm{Nm} / \mathrm{kg})$. The external hip adduction moment increased from $0 \%$ (i.e., right heel contact) to approximately $20 \%$ of the stance phase, but it decreased from approximately $80 \%$ to $100 \%$ (i.e., right toe-off) of the stance phase. Bimodal peaks were also observed in this analysis.

Table 2 presents the results for the analysis of the gait parameters. The mean and standard deviation (SD), minimum, and maximum values for each variable (hip moment impulse in the frontal plane, first peak external hip adduction moment, step length, cadence, walking speed, and stance time) are shown.

Table 3 presents the correlation coefficients between variables (step length, cadence, walking speed, stance time, and hip moment impulse in the frontal plane). 
159

160

161

162

163

164

165

166

167

168

169

170

171

172

173

174

175

176

177

178

179

180

181

182

183

184

185

186

187

188

189

190

191

192

193

194

195

196

197

198

\section{Discussion}

This study examined the effects of step length and cadence on hip moment impulse in the frontal plane during the stance phase. Our main findings are as follows: (1) a decrease in step length decreased the hip moment impulse in the frontal plane, and (2) a decrease in cadence increased the hip moment impulse in the frontal plane. Therefore, our hypotheses were confirmed.

Patients with hip osteoarthritis exhibit decreased walking speed (Constantinou et al., 2017; Foucher, 2017; Meyer et al., 2018; Wesseling et al., 2018; Diamond et al., 2018) and step length (Constantinou et al., 2017). However, walking speed depends on step length and cadence. Although several factors have been reported to affect hip moment impulse in the frontal plane during the stance phase (Inai et al., 2018, 2019a,b; Tateuchi et al., 2020), the effects of step length and cadence on hip moment impulse in the frontal plane are not known to date. Our results help to understand the gait pattern with low hip moment impulse in the frontal plane.

An increase in stride length leads to an increase in the first peak external hip adduction moment during the stance phase (Ardestani et al., 2016). Therefore, we suggest that a decrease in step length may result in a low amplitude of the external hip adduction moment. Furthermore, the hip moment impulse in the frontal plane also decreases because the hip moment impulse in the frontal plane is calculated by the integration of the external hip adduction moment.

Tateuchi et al. (2020) examined the effect of gait kinematics of the hip, pelvis, and trunk on external hip adduction moment through hierarchical multiple regression analyses. They reported that the hip moment impulse in the frontal plane deceased with a decrease in stance time, and body mass and stance time accounted for $61 \%$ of the variance in hip moment impulse in the frontal plane (Tateuchi et al., 2020). Therefore, the effect of stance time on hip moment impulse in the frontal plane is substantial. Additionally, cadence and stance time were significantly negatively correlated in the present study (Table 3). Although the calculation process clarifies that the moment impulse is associated with cadence, that is, stance time because moment impulse is a time-integrated value, our study is the first to examine the effect of cadence on the hip moment impulse in the frontal plane.

In our study (Table 1), the standardized partial regression coefficients of step length and cadence are $0.63(\mathrm{t}=5.24)$ and $-0.60(\mathrm{t}=-4.58)$, respectively. The absolute values of the standardized partial regression coefficients of step length and cadence were the same; therefore, the effects of step length and cadence on hip moment impulse in the frontal plane were similar in the present study. However, the mean walking speed was $1.44(\mathrm{SD}=0.11) \mathrm{m} / \mathrm{s}$ in the present study (mean: $0.82 \mathrm{~m} /(\mathrm{sHT}) \times 1.76 \mathrm{~m}, \mathrm{SD}=0.06 \mathrm{~m} /(\mathrm{sHT}) \times 1.76 \mathrm{~m}$, where HT is height). According to Inai et al. (2019a), the effect of a walking speed of approximately $1.3-1.5 \mathrm{~m} / \mathrm{s}$ on hip moment impulse in the frontal plane was small. In contrast, the effect of walking speed $<1.2$ $\mathrm{m} / \mathrm{s}$ on hip moment impulse in the frontal plane is large, and the hip moment impulse in the frontal plane increased exponentially with a decrease in walking speed (Inai et al., 2019a). 
199

200

201

202

203

204

205

206

207

208

209

210

211

212

213

214

215

216

217

218

219

220

221

222

223

224

225

226

227

228

229

230

231

232

233

234

235

236

237

238

Therefore, the values of the standardized partial regression coefficients of step length and cadence may change in patients with varying slow walking speeds (e.g., absolute values of the standardized partial regression coefficients of step length and cadence are small and large, respectively).

In the present study, the mean hip moment impulse in the frontal plane (not normalized to step length) during walking is $0.50(\mathrm{SD}=0.17) \mathrm{Nm} \mathrm{s} / \mathrm{kg}$. Tateuchi et al. (2017) determined that the mean hip moment impulse in the frontal plane during walking at a self-selected speed was $0.41(\mathrm{SD}=0.13) \mathrm{Nm} / \mathrm{kg}$ (mean: $22.7 \mathrm{Nm} \mathrm{s} / 55.2 \mathrm{~kg}, \mathrm{SD}=7.4 \mathrm{Nm} \mathrm{s} / 55.2 \mathrm{~kg}$ ). Therefore, the value of the hip moment impulse in the frontal plane in the present study is similar to that reported by Tateuchi et al. (2017). Furthermore, the mean first peak external hip adduction moment during the stance phase in the present study was $1.17(\mathrm{SD}=0.32) \mathrm{Nm} / \mathrm{kg}$ (Table 3), which is similar to that reported by Tateuchi et al. $[1.05(\mathrm{SD}=0.29) \mathrm{Nm} / \mathrm{kg}$ (mean: $57.9 \mathrm{Nm} / 55.2 \mathrm{~kg}, \mathrm{SD}=16.0$ $\mathrm{Nm} / 55.2 \mathrm{~kg}$ )] (Tateuchi et al., 2017). Therefore, we believe that the values obtained in the present study are quantitatively reasonable.

This study has a few limitations. First, we used a public dataset, and the participants in the dataset were young (Horst et al., 2019a,b). Chehab et al. (2017) reported that age does not affect the external hip adduction moment. Therefore, the effect of age on hip moment impulse in the frontal plane may be small. Second, the participants in the present study did not have hip osteoarthritis and were healthy. Diamond et al. (2018) reported that the hip joint moments of patients with hip osteoarthritis were different from those of normal participants during walking. Although the results of this study need to be validated in patients with hip osteoarthritis, our findings may be useful in understanding the gait pattern with low hip moment impulse in the frontal plane. The effects of step length and cadence on hip moment impulse in the frontal plane should be further investigated in patients with hip osteoarthritis.

\section{Conclusions}

Our study revealed that a decrease in step length decreased the hip moment impulse in the frontal plane and a decrease in cadence increased the hip moment impulse in the frontal plane. These findings may be useful for understanding gait patterns when the hip moment impulse in the frontal plane is low (or high).

\section{Acknowledgements}

We would like to thank Editage for English language editing.

\section{References}

Ardestani MM, Ferrigno C, Moazen M, Wimmer MA. 2016. From normal to fast walking: Impact of cadence and stride length on lower extremity joint moments. Gait \& Posture 46:118-125. DOI: 10.1016/j.gaitpost.2016.02.005.

Boutron I, Rannou F, Jardinaud-lopez M, Meric G, Revel M, Poiraudeau S. 2008. Disability and quality of life of patients with knee or hip osteoarthritis in the primary care setting and 
239

240

241

242

243

244

245

246

247

248

249

250

251

252

253

254

255

256

257

258

259

260

261

262

263

264

265

266

267

268

269

270

271

272

273

274

275

276

277

278

279

280

281

282

factors associated with general practitioners' indication for prosthetic replacement within 1 year. Osteoarthritis and Cartilage 16:1024-1031. DOI: 10.1016/j.joca.2008.01.001.

Chehab EF, Andriacchi TP, Favre J. 2017. Speed, age, sex, and body mass index provide a rigorous basis for comparing the kinematic and kinetic profiles of the lower extremity during walking. Journal of Biomechanics 58:11-20. DOI: 10.1016/j.jbiomech.2017.04.014.

Cohen J. 1992. A power primer. Psychological bulletin 112:155-159. DOI: 10.1037//00332909.112.1.155.

Constantinou M, Loureiro A, Carty C, Mills P, Barrett R. 2017. Hip joint mechanics during walking in individuals with mild-to-moderate hip osteoarthritis. Gait \& Posture 53:162167. DOI: 10.1016/j.gaitpost.2017.01.017.

Diamond LE, Allison K, Dobson F, Hall M. 2018. Hip joint moments during walking in people with hip osteoarthritis: A systematic review and meta-analysis. Osteoarthritis and Cartilage 26:1415-1424. DOI: 10.1016/j.joca.2018.03.011.

Foucher KC. 2017. Sex-specific hip osteoarthritis-associated gait abnormalities: Alterations in dynamic hip abductor function differ in men and women. Clinical Biomechanics 48:24-29. DOI: 10.1016/j.clinbiomech.2017.07.002.

Holla JFM, van der Leeden M, Roorda LD, Bierma-Zeinstra SMA, Damen J, Dekker J, Steultjens MPM. 2012. Diagnostic accuracy of range of motion measurements in early symptomatic hip and/or knee osteoarthritis. Arthritis Care \& Research 64:59-65. DOI: 10.1002/acr.20645.

Holla JFM, Steultjens MPM, van der Leeden M, Roorda LD, Bierma-Zeinstra SMA, den Broeder AA, Dekker J. 2011. Determinants of range of joint motion in patients with early symptomatic osteoarthritis of the hip and/or knee: An exploratory study in the CHECK cohort. Osteoarthritis and Cartilage 19:411-419. DOI: 10.1016/j.joca.2011.01.013.

Horst F, Lapuschkin S, Samek W, Müller KR, Schöllhorn WI. 2019a. A public dataset of overground walking kinetics and full-body kinematics in healthy individuals. Mendeley Data v2. DOI: 10.17632/svx74xcrjr.2.

Horst F, Lapuschkin S, Samek W, Müller KR, Schöllhorn WI. 2019b. Explaining the unique nature of individual gait patterns with deep learning. Scientific Reports 9. DOI: 10.1038/s41598-019-38748-8.

Iidaka T, Muraki S, Akune T, Oka H, Kodama R, Tanaka S, Kawaguchi H, Nakamura K, Yoshimura N. 2016. Prevalence of radiographic hip osteoarthritis and its association with hip pain in Japanese men and women: The ROAD study. Osteoarthritis and Cartilage 24:117-123. DOI: 10.1016/j.joca.2015.07.017.

Iidaka T, Muraki S, Oka H, Horii C, Kawaguchi H, Nakamura K, Akune T, Tanaka S, Yoshimura N. 2020. Incidence rate and risk factors for radiographic hip osteoarthritis in Japanese men and women: A 10-year follow-up of the ROAD study. Osteoarthritis and Cartilage 28:182-188. DOI: 10.1016/j.joca.2019.09.006.

Inai T, Takabayashi T, Edama M, Kubo M. 2018. Evaluation of factors that affect hip moment impulse during gait: A systematic review. Gait \& posture 61:488-492. DOI: 10.1016/j.gaitpost.2018.02.017.

Inai T, Takabayashi T, Edama M, Kubo M. 2019a. Decrease in walking speed increases hip moment impulse in the frontal plane during the stance phase. PeerJ 7:e8110. DOI: 10.7717/peerj.8110. 
283

284

285

286

287

288

289

290

291

292

293

294

295

296

297

298

299

300

301

302

303

304

305

306

307

308

309

310

311

312

313

314

315

316

317

318

319

320

321

322

323

324

325

326

327

328

Inai T, Takabayashi T, Edama M, Kubo M. 2019b. Effect of contralateral cane use on hip moment impulse in the frontal plane during the stance phase. Gait \& Posture 70:311-316. DOI: 10.1016/j.gaitpost.2019.03.022.

Kito N, Shinkoda K, Yamasaki T, Kanemura N, Anan M, Okanishi N, Ozawa J, Moriyama H. 2010. Contribution of knee adduction moment impulse to pain and disability in Japanese women with medial knee osteoarthritis. Clinical Biomechanics 25:914-919. DOI: 10.1016/j.clinbiomech.2010.06.008.

de Leva P. 1996. Adjustments to Zatsiorsky-Seluyanov's segment inertia parameters. Journal of biomechanics 29:1223-1230. DOI: 10.1016/0021-9290(95)00178-6.

Loureiro A, Constantinou M, Diamond LE, Beck B, Barrett R. 2018. Individuals with mild-tomoderate hip osteoarthritis have lower limb muscle strength and volume deficits. $B M C$ Musculoskeletal Disorders 19. DOI: 10.1186/s12891-018-2230-4.

Loureiro A, Mills PM, Barrett RS. 2013. Muscle weakness in hip osteoarthritis: A systematic review. Arthritis Care \& Research 65:340-352. DOI: 10.1002/acr.21806.

Meyer CAG, Wesseling M, Corten K, Nieuwenhuys A, Monari D, Simon J-P, Jonkers I, Desloovere K. 2018. Hip movement pathomechanics of patients with hip osteoarthritis aim at reducing hip joint loading on the osteoarthritic side. Gait \& posture 59:11-17. DOI: 10.1016/j.gaitpost.2017.09.020.

Pisters MF, Veenhof C, van Dijk GM, Dekker J, CARPA Study Group. 2014. Avoidance of activity and limitations in activities in patients with osteoarthritis of the hip or knee: A 5 year follow-up study on the mediating role of reduced muscle strength. Osteoarthritis and Cartilage 22:171-177. DOI: 10.1016/j.joca.2013.12.007.

Pisters MF, Veenhof C, van Dijk GM, Heymans MW, Twisk JWR, Dekker J. 2012. The course of limitations in activities over 5 years in patients with knee and hip osteoarthritis with moderate functional limitations: Risk factors for future functional decline. Osteoarthritis and Cartilage 20:503-510. DOI: 10.1016/j.joca.2012.02.002.

Robertson DGE, Caldwell GE, Hamill J, Kamen G, Whittlesey SN. 2013. Research Methods in Biomechanics. Champaign: Human Kinetics.

Salaffi F, Carotti M, Stancati A, Grassi W. 2005. Health-related quality of life in older adults with symptomatic hip and knee osteoarthritis: A comparison with matched healthy controls. Aging clinical and experimental research 17:255-263. DOI: 10.1007/bf03324607.

Schmidt A, Meurer A, Lenarz K, Vogt L, Froemel D, Lutz F, Barker J, Stief F. 2017. Unilateral hip osteoarthritis: The effect of compensation strategies and anatomic measurements on frontal plane joint loading. Journal of Orthopaedic Research 35:1764-1773. DOI: 10.1002/jor.23444.

Tateuchi H, Akiyama H, Goto K, So K, Kuroda Y, Ichihashi N. 2020. Gait kinematics of the hip, pelvis, and trunk associated with external hip adduction moment in patients with secondary hip osteoarthritis: Toward determination of the key point in gait modification. $B M C$ Musculoskeletal Disorders 21. DOI: 10.1186/s12891-019-3022-1.

Tateuchi H, Koyama Y, Akiyama H, Goto K, So K, Kuroda Y, Ichihashi N. 2017. Daily cumulative hip moment is associated with radiographic progression of secondary hip osteoarthritis. Osteoarthritis and Cartilage 25:1291-1298. DOI: 10.1016/j.joca.2017.02.796.

Wesseling M, Meyer C, Corten K, Desloovere K, Jonkers I. 2018. Longitudinal joint loading in patients before and up to one year after unilateral total hip arthroplasty. Gait \& posture 61:117-124. DOI: 10.1016/j.gaitpost.2018.01.002.

PeerJ reviewing PDF | (2020:11:55806:4:0:NEW 28 Jun 2021) 
329 Zacharias A, Pizzari T, English DJ, Kapakoulakis T, Green RA. 2016. Hip abductor muscle volume in hip osteoarthritis and matched controls. Osteoarthritis and Cartilage 24:17271735. DOI: 10.1016/j.joca.2016.05.002. 
332 Figure 1. The relationship between step length, cadence, and hip moment impulse in the frontal 333 plane during the stance phase.

334 Red points mean an increased hip moment impulse during the stance phase, and the shade of 335 color indicates the quantitative values of the hip moment impulse in the frontal plane.

336

337 Figure 2. The average wave form of the external hip adduction moment during the stance phase. 338 A bimodal peak was observed. Error bars mean the standard deviations. 


\section{Table 1 (on next page)}

The results of the multiple regression analysis 
Table 1. The results of the multiple regression analysis

\begin{tabular}{lccc}
\hline \multicolumn{1}{c}{ Independent variable } & Standardized partial regression coefficient & $t$ value & $p$ value \\
\hline Step length, $\mathrm{m} / \mathrm{HT}$ & 0.63 & 5.24 & $<0.001$ \\
Cadence, steps/min & -0.60 & -4.58 & $<0.001$ \\
\hline
\end{tabular}

Adjusted $R^{2}=0.71 . \mathrm{F}(2,28)=37.0(p<0.001)$. Dependent variable: hip moment impulse in the frontal plane during the stance phase $(\mathrm{Nms} / \mathrm{kg})$. HT: Height. 
Table 2 (on next page)

Results of the gait parameters 
Table 2. Results of the gait parameters.

\begin{tabular}{|c|c|c|c|c|}
\hline & Mean & SD & Minimum & Maximum \\
\hline Hip moment impulse in the frontal plane, Nms/kg & 0.50 & 0.17 & 0.27 & 0.92 \\
\hline First peak external hip adduction moment, Nm/kg & 1.17 & 0.32 & 0.67 & 1.84 \\
\hline Step length, m/HT & 0.40 & 0.03 & 0.35 & 0.45 \\
\hline Cadence, steps/min & 114.9 & 7.0 & 98.4 & 127.9 \\
\hline Walking speed, m/(sHT) & 0.82 & 0.06 & 0.73 & 0.96 \\
\hline Stance time, $\mathrm{s}$ & 0.61 & 0.05 & 0.53 & 0.73 \\
\hline
\end{tabular}

HT: Height. 
Table 3(on next page)

The correlation coefficients between step length, cadence, walking speed, and stance time 
Table 3. The correlation coefficients between step length, cadence, walking speed, stance time, and hip moment impulse in the frontal pane

\begin{tabular}{|c|c|c|c|c|}
\hline & Cadence & Walking speed & Stance time & $\begin{array}{l}\text { Hip moment impulse in the } \\
\text { frontal plane, Nms/kg }\end{array}$ \\
\hline Step length, m/HT & $r=-0.35, p=0.056$ & $r=0.76, p<0.001$ & $r=0.27, p=0.132$ & $r=0.73, p<0.001$ \\
\hline Cadence, steps/min & - & $r=0.30, p=0.100$ & $r=-0.97, p<0.001$ & $r=-0.65, p<0.001$ \\
\hline Walking speed, m/(sHT) & - & - & $r=-0.37, p=0.040$ & $r=0.35, p=0.058$ \\
\hline Stance time, s & - & - & - & $r=0.57, p<0.001$ \\
\hline
\end{tabular}

$\mathrm{HT}$ : Height. Bold letters indicate a significant difference. 
Figure 1

The relationship between step length, cadence, and hip moment impulse in the frontal plane during the stance phase.

Red points mean an increased hip moment impulse during the stance phase, and the shade of color indicates the quantitative values of the hip moment impulse in the frontal plane.

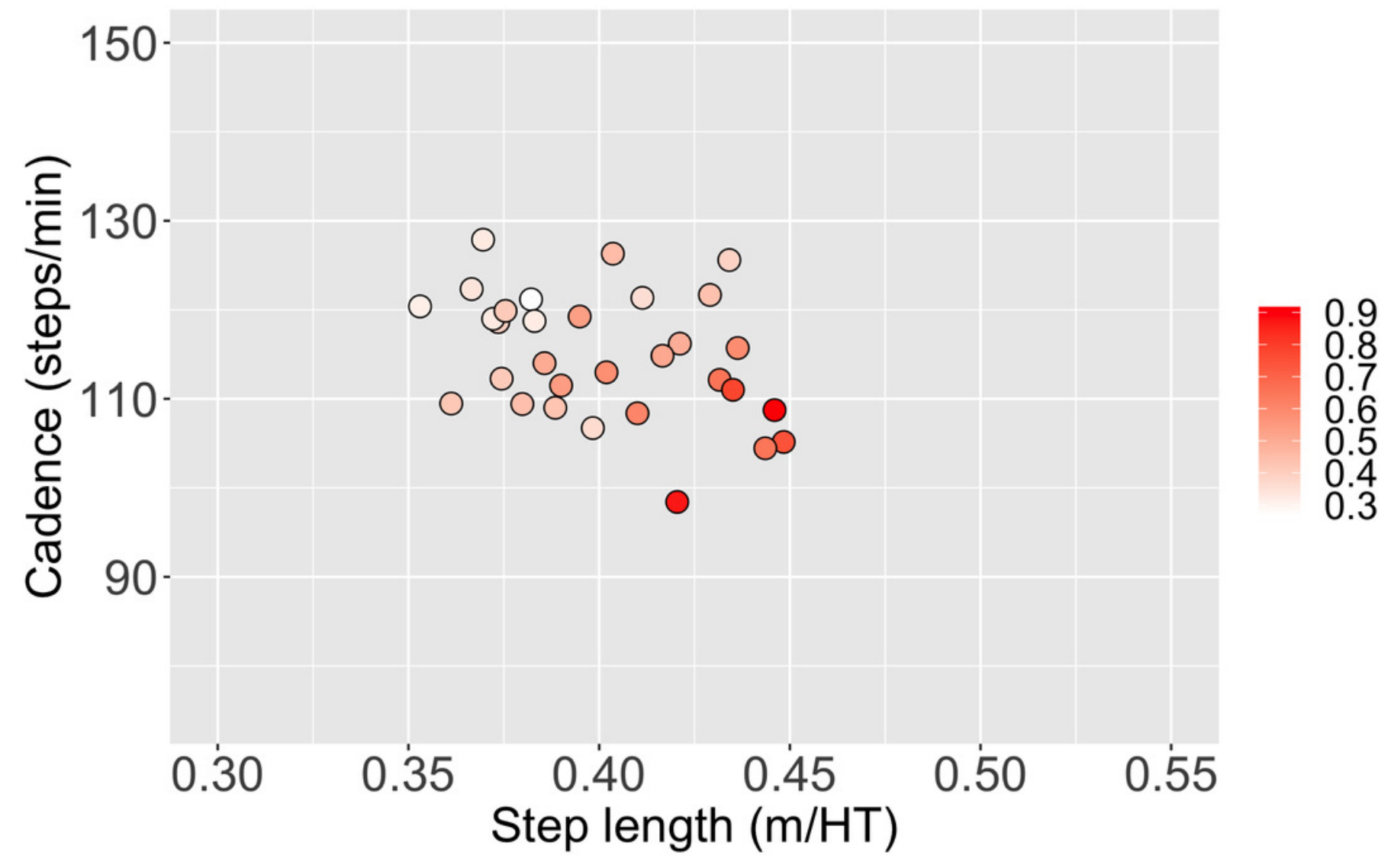


Figure 2

The average wave form of the external hip adduction moment during the stance phase.

A bimodal peak was observed. Error bars mean the standard deviations.

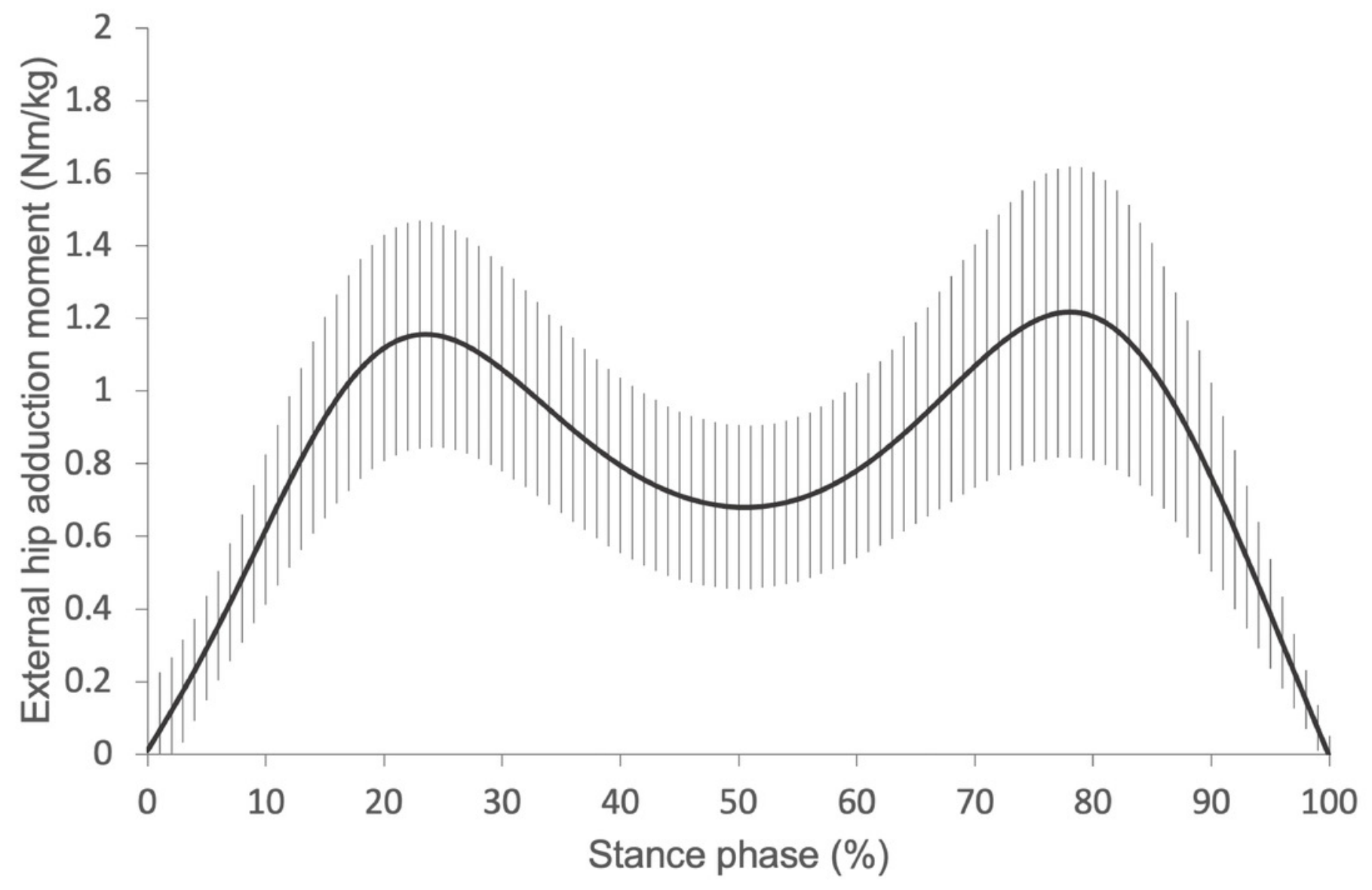

